\title{
Empleabilidad de los egresados del programa de Nutrición y Dietética de una Institución Universitaria
}

\author{
Employability of graduates from the Nutrition and Dietetic program \\ of a University Institution \\ Empregabilidade dos graduados do programa de Nutrição e Dietética \\ da uma Instituição Universitária
}

Fabián Marín' ${ }^{1}$ Brigitt Berdugo ${ }^{1 *}$, Sandra Molina', Erleny Rincón'

Recibido: 25 de junio de 2020. Aceptado para publicación: 10 de septiembre de 2020

Publicado primero en línea: 19 de septiembre de 2020

https://doi.org/10.35454/rncm.v4n1.164

\section{Resumen}

Introducción: en los últimos años la profesión de nutrición y dietética ha tenido gran demanda y aceptación debido a que las personas son cada vez más conscientes de la relación directa entre salud y alimentación; sin embargo, es poco lo que está documentado sobre la ocupación laboral de los egresados de esta profesión.

Objetivo: caracterizar los aspectos laborales y ocupacionales de los graduados del programa de Nutrición y Dietética de la Institución Universitaria Escuela Nacional del Deporte.

Métodos: es un estudio descriptivo de enfoque cuantitativo y corte transversal. Se realizó la Encuesta de Seguimiento a Graduados del Ministerio de Educación del 2017, a través del formulario Google formulario en modalidad online a una muestra de 41 egresados entre 2017 y 2019.

Resultados: $98 \%$ de los egresados son mujeres. En la actualidad $95 \%$ son activos laboralmente, $80 \%$ vinculados en el sector privado y $20 \%$ en el sector público, con una contratación a término indefinido de $46 \%$, seguido por prestación de servicio en $34 \%$. El área con mayor ocupación es la consulta externa (39\%) y la clínica (34\%). El $12 \%$ tiene más de un empleo y los salarios oscilan entre 2 a 3 SMLV en $76 \%$ de la población estudio.

\section{Summary}

Introduction: The profession of nutrition and dietetics has been in great demand and acceptance in recent years due to the fact that people are increasingly aware of the direct relationship between health and nutrition. However, there is scarce documentation on the occupancy rate of graduates in this profession.

Objective: The purpose of the study was to describe employment and occupancy aspects of graduates from the Nutrition and Dietetics program of the IU. National School of Sports.

Methods: This was a descriptive, quantitative, cross-sectional study. Through an online Google form, we conducted a followup survey on graduates from the Ministry of Education between 2017 and 2019.

Results: From 41 responders, 98\% were women and $2 \%$ were men. At that moment, $95 \%$ of graduates were actively employed, $80 \%$ in the private sector and $20 \%$ in the public sector, with $46 \%$ having a permanent contract, and 34\% having a contract for provision of services. The areas with the highest occupancy were outpatient (39\%) and clinic (34\%). Twelve percent had more than one job, and in $76 \%$ of the study population salaries ranged between 2 to 3 current minimum legal wages.

\section{Resumo}

Introdução: nos últimos anos, a profissão de nutrição e dietética tem tido grande procura e aceitação devido ao fato de que as pessoas estão cada vez mais conscientes da relação direta entre saúde e alimentação; no entanto, pouco está documentado sobre a ocupação profissional dos ingressados nesta profissão.

Objetivo: caracterizar os aspetos laborais e ocupacionais dos ingressados no programa de Nutrição e Dietética da Institución Universitaria Escuela Nacional del Deporte.

Métodos: é um estudo descritivo com abordagem quantitativa e transversal. A Pesquisa de Acompanhamento de Pós-Graduação 2017 do Ministério da Educação foi realizada por meio do formulário do Google na modalidade online para uma amostra de 41 graduados entre 2017 e 2019.

Resultados: $98 \%$ dos graduados são mulheres. Atualmente 95\% estão ativos com emprego, $80 \%$ vinculados ao setor privado e $20 \%$ ao setor público, com contrato por prazo indeterminado de $46 \%$, seguido da prestação de serviços em $34 \%$. A área de maior ocupação é a consulta externa (39\%) e a clínica (34\%). 12\% têm mais de um emprego e os salários variam de 2 a 3 salario mínimo em $76 \%$ da população estudada. 
Conclusiones: existe una baja tasa de desempleo (5 \%) y una fuente de trabajo alta en el Valle del Cauca para la carrera de nutrición, en áreas asistenciales y clínicas. A pesar de que la modalidad de prestación de servicios puntea en la actualidad en nuestro país; aún se conservan contratos a término indefinido.

Palabras claves: graduado, nutricionista, empleo.
Conclusions: In Valle del Cauca unemployment rate is low (5\%), and there is a high source of employment for nutritionists in healthcare and clinical areas. Although the provision of services model is gaining momentum in our country, permanent contracts are still the norm.

Keywords: Graduate; Nutritionist; Job.
Conclusões: existe uma baixa taxa de desemprego (5\%) e uma elevada fonte de empregos no Vale do Cauca para a carreira de nutrição, saúde e clínica. Apesar de a modalidade de prestação de serviços atualmente se destacar em nosso país; contratos por prazo indeterminado ainda são mantidos.

Palavras-chave: pós-graduação, nutricionista, emprego.
Oficina de Egresados. Institución Universitaria Escuela Nacional del Deporte, Cali, Valle del Cauca, Colombia.
*Correspondencia: Brigitt Berdugo brigitt.berdugo@endeporte.edu.co

\section{INTRODUCCIÓN}

La Nutrición y Dietética como ciencia del área de la salud estudia las funciones vitales y el manejo de condiciones fisiológicas específicas del proceso saludenfermedad del ser humano en lo individual y social. En el contexto internacional latinoamericano, la formación de profesionales en Nutrición y Dietética fue el resultado de la necesidad de contar con personal capacitado en dietética hospitalaria, con un enfoque principalmente terapéutico como personal de apoyo en la preparación y suministro de dietas para el tratamiento de diferentes enfermedades. En 1933 el doctor Pedro Escudero creó la primera Escuela de Dietistas, adscrita a la Escuela de Medicina de la Universidad en Buenos Aires, Argentina. En distintos países de América del Sur y Centroamérica se crearon otros programas, denominándose escuelas o facultades de Nutrición o de Dietética según su orientación ${ }^{(1)}$. El desempeño profesional del nutricionista dietista colombiano ha evolucionado ampliando su rol a diversos campos de acción y gestión; el Congreso de Colombia, por la Ley 73 de 1979 Reglamentó el ejercicio de la profesión de Nutrición y Dietética ${ }^{(2)}$ y la Asociación Colombiana de Dietistas y Nutricionistas (ACODIN), definió el perfil profesional del Nutricionista Dietista en el Código de Bioética en el año $1999^{(3)}$; este código es la normatividad vigente para el desempeño profesional.

Según la Asociación Colombiana de Facultades de Nutrición y Dietética (ACOFANUD), en 2013 había 8.905 egresados en todo el país ${ }^{(1)}$. En este mismo año nace el programa de nutrición y dietética de la IU Escuela Nacional del Deporte debido a la alta oferta y necesidad de profesionales en esta área en el suroccidente colombiano; tuvo su primera promoción en 2017 y hasta 2019 han egresado 143 profesionales.

Hoy en día el rol del nutricionista es importante por su intervención en algunas problemáticas relacionadas con el estado nutricional en el país como la doble carga nutricional. De acuerdo con la Encuesta Nacional de la Situación Alimentaria de 2015 (ENSIN) ${ }^{(4)}$, en Colombia $54,2 \%$ de los hogares se encuentran en inseguridad alimentaria, y 10,8 \% menores de 5 años con retraso en la talla, por otro lado adolescentes y adultos presentan exceso de peso ( $17,9 \%$ y $56,4 \%$ respectivamente), por tal motivo existen grandes desafíos para mejorar esta situación.

Teniendo en cuenta que los profesionales de esta importante rama de la salud suman esfuerzos para combatir las situaciones antes mencionadas, se hace cada vez más importante para las universidades, iniciar y mantener programas de seguimiento a egresados, que aporten información sobre aspectos sociodemográficos, desempeño laboral, ocupacional y profesional.

Por otra parte, la Ley 1164 de octubre de $2007^{(5)}$, dicta disposiciones en materia del Talento Humano en Salud haciendo énfasis en la vigilancia y control del ejercicio, así como de su desempeño profesional. El Ministerio de Educación a través del Observatorio Laboral para la Educación ${ }^{(6)}$, en sus resultados evidencia que la carrera de Nutrición y Dietética no hace parte del ranking de profesiones con mayor índice de empleabilidad, situación que ratifica la importancia de hacer seguimiento a egresados que arroje datos que permitan conocer el desempeño laboral y que contribuya a identificar áreas que no están siendo cubiertas por la oferta actual de pro- 
fesionales, información que puede resultar valiosa, para la toma de decisiones de los actores involucrados en el proceso educativo y futuros empleadores.

Por lo anterior, el propósito de este estudio fue caracterizar los aspectos laborales y ocupacionales de los egresados del programa de pregrado en Nutrición y Dietética de la Institución Universitaria Escuela Nacional del Deporte.

\section{MATERIALES Y MÉTODOS}

Es un estudio descriptivo de enfoque cuantitativo y corte transversal con un universo de 143 estudiantes graduados entre el año 2017 y 2019 del programa de Nutrición y Dietética de la Institución Universitaria Escuela Nacional del Deporte. La recopilación de los datos se hizo a través de la oficina de egresados de la institución, a partir de la base de datos de los graduados. Se aplicó el instrumento en modalidad online a través de la herramienta Google Formulario, enviando el enlace al correo electrónico del profesional con la Encuesta de Seguimiento a Graduados V 1.0-2017 del Ministerio de Educación ${ }^{(7)}$ que analiza el desarrollo profesional y personal de los graduados de educación superior; visualiza nuevas perspectivas para complementar su proceso de formación y el acceso al mercado laboral. Después del envío de la encuesta se realizaron tres recordatorios por correo electrónico; la encuesta se mantuvo abierta durante 30 días.

Este instrumento consta de cinco partes: información personal y familiar; competencias; plan de vida; situación laboral y nivel de identidad con la Institución de Educación Superior.

\section{Aspectos éticos}

Los procedimientos realizados en este estudio no implicaron riesgo o perjuicios para los egresados. Previo al inicio de la encuesta debían firmar el consentimiento informado y la autorización para el uso de los datos.

El proyecto fue avalado por el Comité de Ética de Investigación en humanos de la Institución Universitaria Escuela Nacional del Deporte, el formato contó con la estructura establecida por el Ministerio de Protección Social mediante la Resolución 8430 de 1993 de la de Colombia ${ }^{(8)}$.

\section{Análisis estadístico}

El procesamiento de la información de las encuestas se utilizó el programa estadístico SPSS V. 22,0 (IBM
SPSS Statistics, Armonk, NY, EEUU). A través de la prueba de chi-cuadrado de Pearson (X2) se estableció la existencia de relaciones estadísticamente significativas entre el tipo de contratación y área de desempeño, salario y área de desempeño, tipo de contrato y salario.

\section{RESULTADOS}

La encuesta fue enviada a todos los egresados; sin embargo, solo 30 \% (41 egresados) la contestó y completó correctamente, que es lo mínimo recomendado para no caer en la categoría de muestra pequeña ${ }^{(9)}$.

\section{Características sociodemográficas}

$98 \%$ de los graduados son mujeres y $2 \%$ hombres; en edades que oscilan entre los 20 a 25 años $(61 \%)$ y residentes en su mayoría en la ciudad de Cali (78\%) (Tabla 1).

Tabla 1. Características sociodemográficas

\begin{tabular}{|c|c|c|c|}
\hline \multicolumn{2}{|r|}{ Variables } & $\begin{array}{c}\text { Frecuencia } \\
\quad(n=41)\end{array}$ & $\begin{array}{c}\text { Porcentaje } \\
\text { (\%) }\end{array}$ \\
\hline \multirow{2}{*}{ Sexo } & Femenino & 40 & 98 \\
\hline & Masculino & 1 & 2 \\
\hline \multirow{3}{*}{$\begin{array}{l}\text { Rango de } \\
\text { edad }\end{array}$} & 20 a 25 años & 25 & 61 \\
\hline & 25 a 30 años & 14 & 34 \\
\hline & 30 a 35 años & 2 & 5 \\
\hline \multirow{6}{*}{$\begin{array}{l}\text { Ciudad de } \\
\text { residencia }\end{array}$} & Buga & 1 & 2,4 \\
\hline & Cali & 32 & 78 \\
\hline & Jamundí & 1 & 2,4 \\
\hline & Palmira & 1 & 2,4 \\
\hline & $\begin{array}{l}\text { Santander de } \\
\text { Quilichao }\end{array}$ & 5 & 12 \\
\hline & Tuluá & 1 & 2,4 \\
\hline
\end{tabular}

\section{Perfil laboral}

$95 \%$ de la población encuestada, correspondiente a 39 de ellos, están activos laboralmente como nutricionistas dietistas, $5 \%$ no se encuentra laborando; vinculados en $80 \%$ al sector privado y $20 \%$ al sector público. Las instituciones donde laboran son Instituciones Prestadoras de Servicios de Salud (IPS) 34 \% y clínicas $22 \%$ (Tabla 2).

Por otro lado, el contrato a término indefinido prima con $46 \%$, seguido del contrato por prestación de servicios con $34 \%$. Ochenta por ciento de las contratacio- 
Tabla 2. Instituciones donde laboran

\begin{tabular}{|l|l|c|c|}
\hline \multicolumn{2}{|c|}{ Variables } & $\begin{array}{c}\text { Frecuencia } \\
(\mathbf{n = 4 1 )}\end{array}$ & $\begin{array}{c}\text { Porcentaje } \\
\text { (\%) }\end{array}$ \\
\hline \multirow{4}{*}{$\begin{array}{l}\text { Tipo de } \\
\text { institución }\end{array}$} & Privada & 33 & 80 \\
\cline { 2 - 4 } & Pública & 8 & 20 \\
\hline Instituciones & Clínicas & 9 & 22 \\
\cline { 2 - 4 } & Hospitales & 3 & 7 \\
\cline { 2 - 4 } & IPS & 14 & 34 \\
\cline { 2 - 4 } & EPS & 4 & 10 \\
\cline { 2 - 4 } & Fundaciones & 3 & 7 \\
\cline { 2 - 4 } & S.A- Industria & 2 & 5 \\
\cline { 2 - 4 } & $\begin{array}{l}\text { Laboratorios } \\
\text { farmacéuticos }\end{array}$ & 2 & 5 \\
\cline { 2 - 4 } & $\begin{array}{l}\text { Clubes } \\
\text { deportivos }\end{array}$ & 2 & 5 \\
\hline
\end{tabular}

nes de clínicas e IPS son a término indefinido. Por otro lado, $76 \%$ de los salarios oscilan entre 2 y 3 Salarios Mínimos Legales Vigentes (SMLV); y $12 \%$ tiene más de un empleo (Tabla 3). Para el año 2020 un SMLV corresponde a la suma de ochocientos setenta y siete mil ochocientos tres pesos (\$877.803 [USD \$240]) $)^{(10)}$.

$\mathrm{El}$ área de desempeño con mayor cobertura es la consulta externa $39 \%$ con contrato por prestación de servicio de $17 \%$, contrato a término indefinido $17 \%$. La segunda área de desempeño es la clínica (34\%) con predominancia del contrato indefinido de $22 \%$ (Figura 1).

Por otra parte, los salarios más altos se encuentran en las áreas de desempeño de salud pública, deportiva y visita médica; en el campo clínico y asistencial pondera entre 2 y 3 SMLV con $32 \%$ en cada área (Figura 2). En relación con el tipo de contrato y salario, se puede apreciar en la Figura 3, que el contrato indefinido presenta un rango predominante de $46 \%$ entre 2 - 3 SMLV.

Se encontró la existencia de relaciones estadísticamente significativas $(p<0,001)$ entre el tipo de contratación y área de desempeño, salario y área de desempeño, tipo de contrato y salario.

En cuanto al nivel de estudios, $14 \%$ tiene estudios de posgrado en donde $7 \%$ son especialistas y $7 \%$ estudios de maestría (Figura 4).

\section{DISCUSIÓN}

El Consejo Nacional de Acreditación (CNA) refiere en los Lineamientos para la Acreditación de Programas de
Tabla 3. Tipo de contrato

\begin{tabular}{|c|c|c|c|}
\hline \multicolumn{2}{|c|}{ Variables } & \multirow{2}{*}{$\begin{array}{c}\begin{array}{c}\text { Frecuencia } \\
(\mathbf{n}=\mathbf{4 1})\end{array} \\
2\end{array}$} & \multirow{2}{*}{$\begin{array}{c}\begin{array}{c}\text { Porcentaje } \\
\text { (\%) }\end{array} \\
5\end{array}$} \\
\hline Labora & No & & \\
\hline 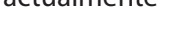 & $\mathrm{Si}$ & 39 & 95 \\
\hline \multirow[t]{2}{*}{ + de 1 empleo } & No & 36 & 88 \\
\hline & $\mathrm{Si}$ & 5 & 12 \\
\hline \multirow[t]{4}{*}{$\begin{array}{l}\text { Tipo de } \\
\text { contrato }\end{array}$} & $\begin{array}{l}\text { Contrato } \\
\text { definido }\end{array}$ & 6 & 15 \\
\hline & $\begin{array}{l}\text { Contrato } \\
\text { indefinido }\end{array}$ & 19 & 46 \\
\hline & $\begin{array}{l}\text { Prestación de } \\
\text { servicios }\end{array}$ & 14 & 34 \\
\hline & No aplica & 2 & 5 \\
\hline \multirow[t]{7}{*}{$\begin{array}{l}\text { Área de } \\
\text { desempeño }\end{array}$} & $\begin{array}{l}\text { Consulta } \\
\text { externa }\end{array}$ & 16 & 39 \\
\hline & Clínica & 14 & 34 \\
\hline & $\begin{array}{l}\text { Nutrición } \\
\text { pública }\end{array}$ & 3 & 7 \\
\hline & $\begin{array}{l}\text { Servicios de } \\
\text { alimentos e } \\
\text { industria }\end{array}$ & 2 & 5 \\
\hline & $\begin{array}{l}\text { Nutrición y } \\
\text { deporte }\end{array}$ & 2 & 5 \\
\hline & Visita médica & 2 & 5 \\
\hline & No aplica & 2 & 5 \\
\hline \multirow[t]{5}{*}{$\begin{array}{l}\text { Salario y } \\
\text { remuneración }\end{array}$} & $\begin{array}{l}\text { Entre } 1 \text { y } 2 \\
\text { SMLV }\end{array}$ & 3 & 7 \\
\hline & 2 a 3 SMLV & 31 & 76 \\
\hline & 3 a 4 SMLV & 3 & 7 \\
\hline & + de 5 SMLV & 2 & 5 \\
\hline & No aplica & 2 & 5 \\
\hline
\end{tabular}

Pregrado, característica 36 de $2013^{(11)}$, que las instituciones de educación superior deben realizar programas que hagan seguimiento a la ubicación y a las actividades que desarrollan los egresados en asuntos concernientes al logro de los fines de la institución y del programa. Esto va en articulación con el observatorio laboral para la educación del Ministerio de Educación quienes solicitan tener esta información ya que permite monitorear el mercado de los egresados y generar conocimiento más amplio para 


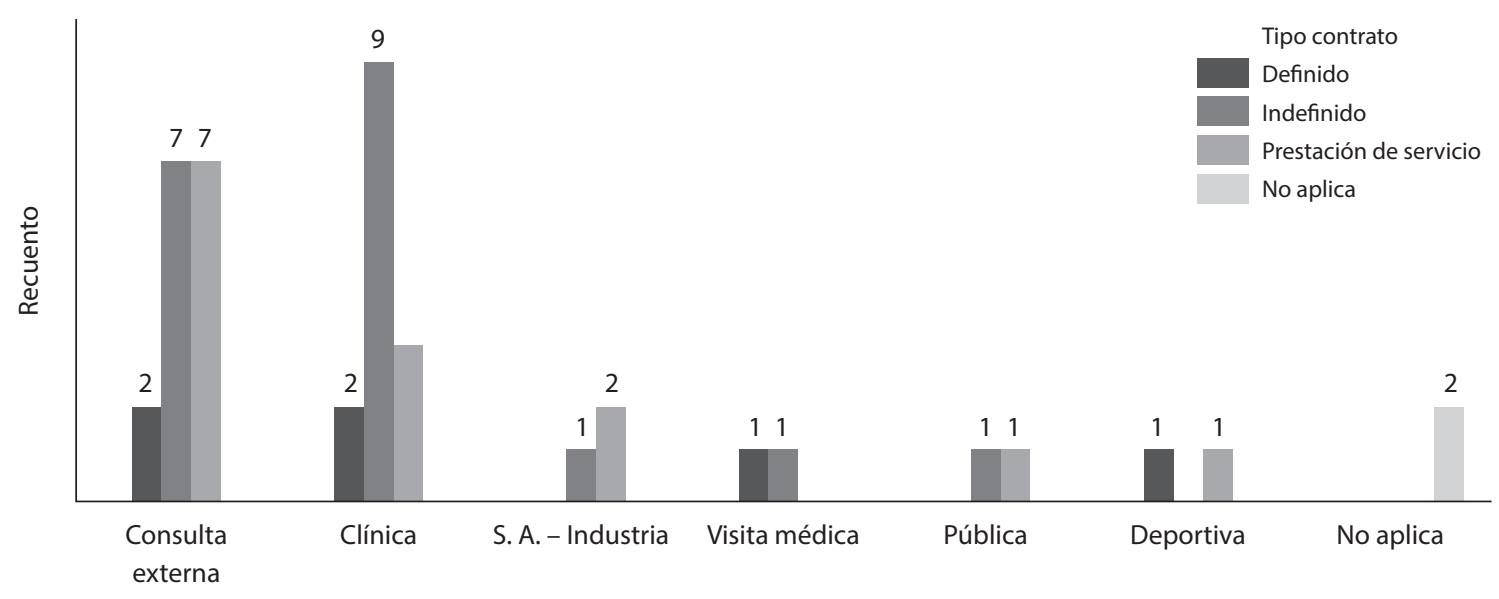

Área de desempeño

Figura 1. Tipos de contratación según el área de desempeño de los egresados encuestados.

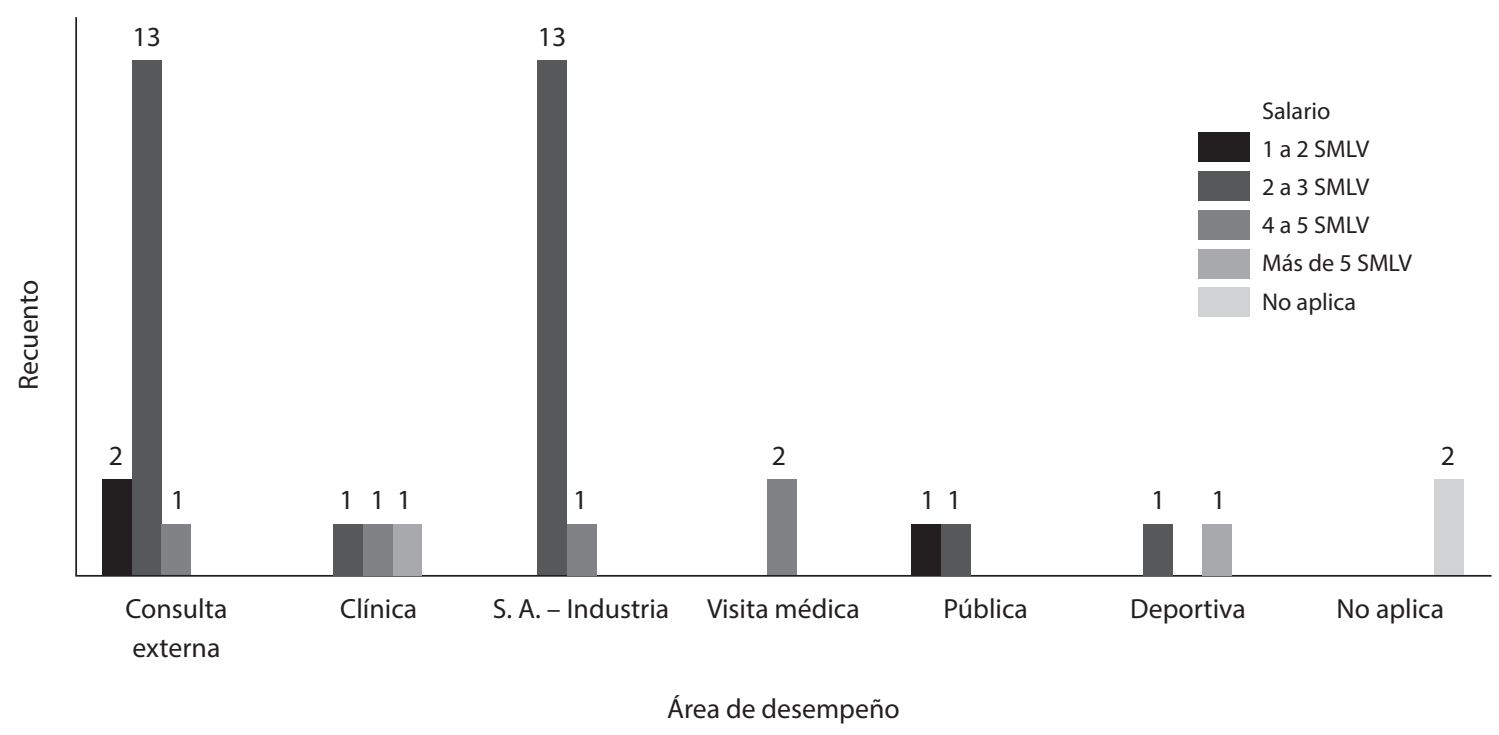

Figura 2. Salario según el área de desempeño de los egresados encuestados.

satisfacer las necesidades de sus diferentes usuarios ${ }^{(12)}$. El principal resultado de este estudio muestra que $95 \%$ de los egresados del programa de nutrición y dietética tiene empleo, lo cual se encuentra alineado con el estudio de ACOFANUD $^{(1)}$. Además, que es una profesión ejercida mayoritariamente por mujeres $65 \%$ - $98 \%{ }^{(1,13,14)}$, y gran parte de este gremio desarrolla su ejercicio profesional en el sector privado ${ }^{(1,14)}$, diferente al estudio realizado en Ecuador donde $76 \%$ está en el sector público ${ }^{(14)}$.

El área con mayor desempeño en este estudio fue la consulta externa; sin embargo, el ejercicio en el área pública ha predominado en esta profesión ${ }^{(1,13,14)}$. Se corrobora que el segundo campo de mayor desempeño es la nutrición clínica; las instituciones que más contratan son las clínicas e IPS ${ }^{(12)}$; y se estableció que $12 \%$ tiene un segundo empleo ${ }^{(13)}$.

Respecto al tipo de contratación de los profesionales participantes de la investigación, se encontró que el contrato a término indefinido predomina en las áreas de desempeño clínico y de consulta externa, presentando un comportamiento similar a lo encontrado en la encuesta de las condiciones laborales en el sector salud con una contratación indefinida para los nutricionistas de $78,6 \%^{(14)}$. Este tipo de contratación hace que 


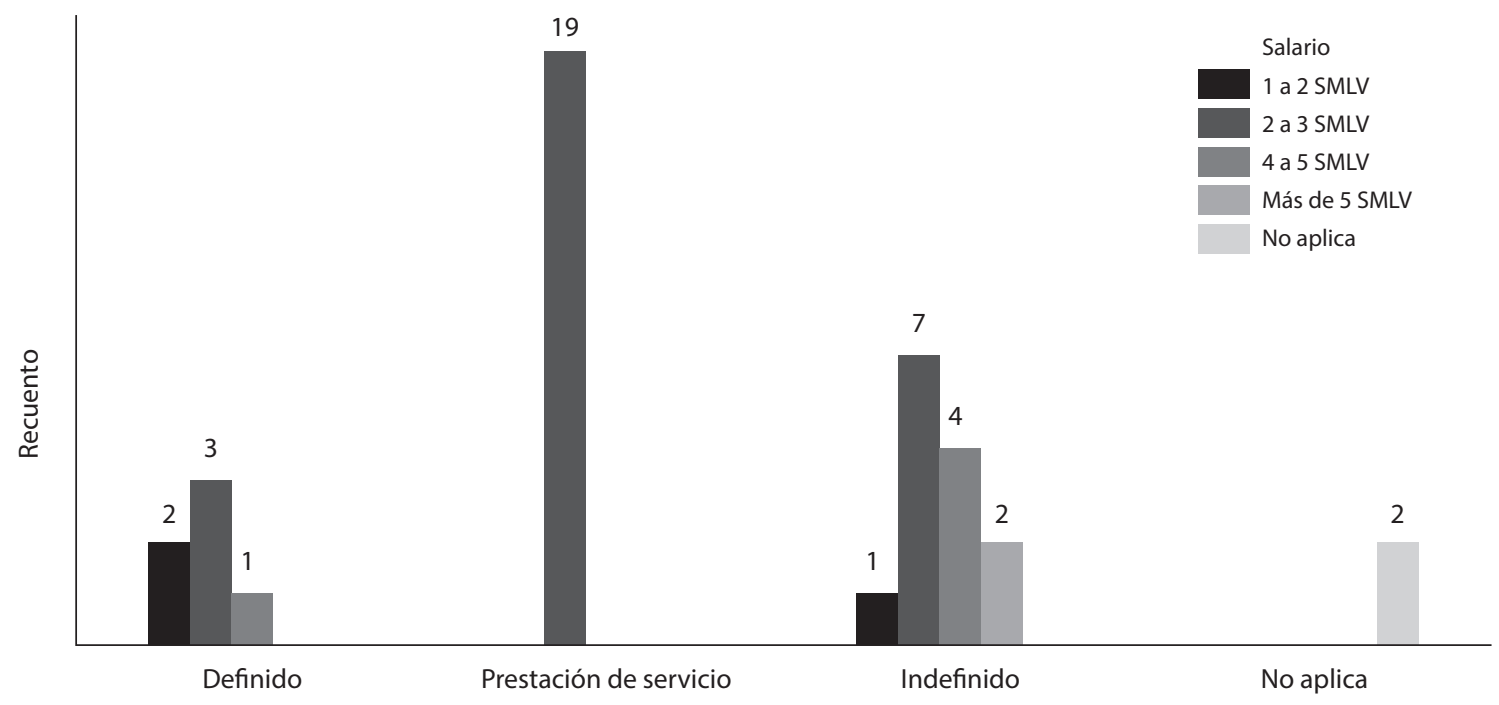

Tipo de contrato

Figura 3. Relación entre tipo de contrato y salario de los egresados encuestados.

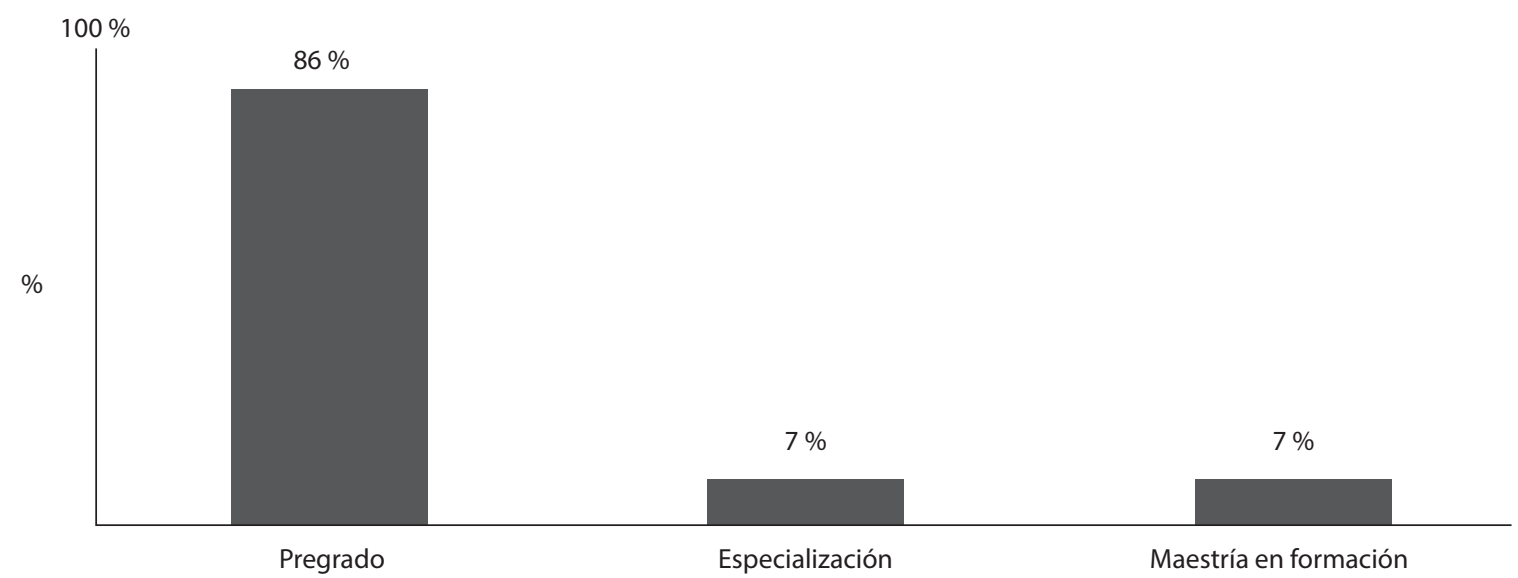

Figura 4. Nivel de estudios de los egresados encuestados.

el monto salarial efectivo recibido por los profesionales de dicha área sea menor en comparación con lo recibido por los nutricionistas de otras áreas de desempeño como en nutrición comunitaria donde reciben salarios mayores, pero corresponde a salarios integrales con contratos por prestación de servicio ${ }^{(15)}$ donde el profesional asume la seguridad social.

Esta información es valiosa para asociaciones gremiales como la Asociación Colombiana de Dietistas y Nutricionistas ACODIN, que dentro de sus objetivos está favorecer el empleo y la remuneración justa y equitativa de los Nutricionistas Dietistas, además de propender por el mejoramiento de las condiciones laborales para el gremio.

Por otra parte, los egresados son en su mayoría adultos jóvenes menores de 30 años y quienes refieren no haber continuado estudios de posgrado ( $88 \%)$, diferente al compararlo con el estudio de la Universidad Javeriana donde $34 \%$ está entre los 20 y 30 años y $40 \%$ ya ha realizado estudios de posgrado ${ }^{(16)}$.

El rango de ingresos está por debajo de lo ponderado para esta profesión según lo devengado por los profesionales nutricionistas dietistas del país, que está entre 3 a $4 \operatorname{SMLV}^{(17)}$. 
Como resultado relevante al comparar la tasa de desempleo con el DANE la carrera de nutrición se encuentra $5 \%$ por debajo de las estadísticas nacionales actuales de $23,5 \%^{(18)}$, un hecho que es favorable para los futuros profesionales.

Una de las limitantes del estudio es la baja participación de los egresados lo que impide hacer referencia a toda la población de nutricionistas del Valle del Cauca, en especial la de los Nutricionistas Dietista graduados de la Institución Universitaria Escuela Nacional del Deporte. Se buscará en el futuro la participación de todos los egresados en este tipo de encuestas.

\section{CONCLUSIONES}

- La mayoría de la población del estudio ejerce su profesión con una tasa de desempleo baja.

- Los graduados en su mayoría están empleados por instituciones privadas; sin embargo, no se observan emprendimientos o generación de empresas.

- La educación continuada como posgrado no hace parte aún de los proyectos a corto plazo de los egresados.

- Se recomienda ampliar este estudio a todos los nutricionistas dietistas que laboran en el Valle del Cauca.

\section{Agradecimientos}

Esta investigación fue desarrollada gracias al apoyo que brindó la coordinadora del programa de nutrición y dietética Alba Leonor Piñeros y los egresados del programa de Nutrición y Dietética de la Institución Universitaria Escuela Nacional del Deporte.

\section{Conflicto de intereses}

Declaramos no tener ningún conflicto de interés.

\section{Financiación}

El presente trabajo no tuvo financiacion.

\section{Declaración de autoria}

F.M. participó en la concepción y obtención de datos; B.B. participó en el desarrollo de la investigación, análisis de datos y redacción del artículo; S.M. participó en la realización de la investigación, en la obtención y análisis de datos; E.R. participó en la interpretación de resultados y redacción del artículo. Todos los autores revisaron el artículo y validaron su versión final.

\section{Referencias bibliográficas}

1. Asociación Colombiana de Facultades de Nutrición y Dietética (ACOFANUD), Asociación Colombiana de Dietistas y Nutricionistas (ACODIN), Comisión de Ejercicio Profesional de Nutrición y Dietética. Perfil y competencias profesionales del nutricionista dietista en Colombia-2013 [Internet]. Bogotá; diciembre de 2013 [Fecha de consulta: 02 de noviembre de 2019]. Disponible en: https://www. minsalud.gov.co/sites/rid/Lists/BibliotecaDigital/RIDE/ VS/TH/Nutricion\%20y\%20Dietetica_Octubre2014.pdf.

2. Ley 73 de 1979 [Internet]. Bogotá D.C. República de Colombia; 28 de diciembre de 1979 [Fecha de consulta: 19 de noviembre de 2019]. Disponible en: https://www.funcionpublica.gov.co/eva/gestornormativo/norma_pdf.php?i=66182.

3. García UA, Carrasco DGC. Código de Bioética [Internet]. Bogotá; D.C; enero de 2003. [Fecha de consulta: 05 de febrero de 2020]. Disponible en: https://studylib.es/doc/8149269/ c\%C3\%B3digo-de-bio\%C3\%A9tica---comisi\%C3\%B3n-deejercicio-profesional-de...

4. Ministerio de Protección Social, Instituto Colombiano de Bienestar Familiar (ICBF), Instituto Nacional de Salud, Universidad Nacional De Colombia. Encuesta Nacional de la Situación Nutricional ENSIN. Colombia; [Internet]. 2015. [Fecha de consulta: 17 febrero 2020]. Disponible en:

5. https://www.nocomasmasmentiras.org/wp-content/ uploads/2017/12/Resultados-ENSIN-2015.pdf.

6. Ley 1164 de 2007 [Internet]. Bogotá D.C. Republica de Colombia; 3 de octubre de 2007. [Fecha de consulta: 05 de febrero de 2020]. Disponible en: https://www.minsalud.gov.co/ Normatividad_Nuevo/LEY\%201164\%20DE\%202007.pdf.

7. Ministerio de Educación. Observatorio Laboral para la Educación. Mirada regional de programas académicos de pregrado destacados por la mayor cantidad de graduados que cotizan al sector formal de la economía y mejores salarios. [Internet]. Bogotá D.C. 15 de enero 2020 [Fecha de consulta: 17 febrero 2020]. Disponible en: https://ole.mineducacion. gov.co/1769/articles-392282_recurso_1.pdf.

8. MINEDUCACION. Encuesta De Seguimiento a Graduados de Educación Superior en el nivel de pregrado V 1.0-2017. [Internet]. Bogotá D.C. 2017 [Fecha de consulta: 18 febrero 2020]. Disponible en: https://encuestasole.mineducacion. gov.co/hecaa-encuestas/inicio.

9. Resolución 8430 de 1993[Internet]. Bogotá D.C. 4 de octubre de 1993. [Fecha de consulta: 6 de febrero de 2020]. Disponible en: https://www.minsalud.gov.co/sites/rid/ Lists/BibliotecaDigital/RIDE/DE/DIJ/RESOLUCION8430-DE-1993.PDF.

10. Pineda EB, de Alvarado El, de Canales FH. Metodología de la investigación [Internet]. Washington: Organización Panamericana de la Salud, Organización Mundial de la Salud; 1994. [Fecha de consulta: 20 marzo 2020]. 111p. Disponible en: http://187.191.86.244/rceis/registro/Metodologia\%20 
de\%20la\%20Investigacion\%20Manual\%20para\%20el\%20 Desarrollo\%20de\%20Personal\%20de\%20Salud.pdf.

11. Decreto 2360 de 2019[Internet]. Bogotá D.C. República de Colombia; 26 diciembre 2019. [Fecha de consulta: 26 de julio de 2020]. Disponible en: https://dapre.presidencia. gov.co/normativa/normativa/DECRETO\%202360\%20\%20 DEL\%2026\%20DICIEMBRE\%20DE\%202019.pdf.

12. Consejo Nacional de Acreditación (CNA). Lineamientos Para La Acreditación De Programas De Pregrado [Internet]. Bogotá D.C. enero de 2013. [Fecha de consulta: 06 de febrero de 2020]. Disponible en: https://www.cna.gov.co/1741/articles-186359_pregrado_2013.pdf.

13. Ministerio de Educación, Sistema Nacional de Información de la Educación Superior. Misión y visión del Observatorio Laboral para la Educación [Internet]. 2020. [Fecha de consulta: 06 de febrero de 2020]. Disponible en: https://ole. mineducacion.gov.co/portal/El-Observatorio/Mision-yvision/.

14. Ajila MAK, Méndez EP. Seguimiento A Graduados De La Carrera De Nutrición Y Salud Comunitaria De La Universidad Técnica Del Norte Del Periodo 2009-2014. [Internet] [Tesis de Licenciatura]. Ibarra: Universidad Técnica Del Norte; 2016. [Fecha de consulta: el 10 de noviembre de 2019]. Disponible en: http://repositorio.utn.edu.ec/bitstream/123456789/6125/1/06\%20NUT\%20191\%20 TRABAJO\%20GRADO.pdf.

15. Florez J, Atehortua S, Arenas A. Las condiciones laborales de los profesionales de la salud a partir de la Ley 100 de 1993: evolución y un estudio de caso para Medellín. Rev. Gerenc. Polit. Salud. [Internet]. 2009. [Fecha de consulta: 04 de septiembre de 2020]; 8 (16). Disponible en: http://www.scielo. org.co/pdf/rgps/v8n16/v8n16a07.pdf.

16. Miranda VA. Caracterización del perfil profesional y laboral de los egresados de la carrera de nutrición y dietética de la pontificia universidad javeriana sede Bogotá, D.C. [Internet] [Tesis de pregrado]. Bogotá D.C.: Universidad Pontificia Universidad Javeriana; 2018. [Fecha de consulta: 20 de noviembre de 2019]. Disponible en: https://repository.javeriana.edu.co/bitstream/handle/10554/39268/ Caracterizaci\%C3\%B3n\%20del\%20perfil\%20profesional\%20 y\%20laboral\%20de\%20los\%20egresados\%20de\%20la\%20 Carrera\%20de\%20Nutrici\%C3\%B3n\%20y\%20Diet\%C3\%A9tica $\% 20$ de $\% 201$ a $\% 20$ Pontificia $\% 20$ Universidad $\% 20$ Javeriana\%20sede\%20Bogot\%C3\%A1\%20DC\%20\%20 Arturo $\% 20$ Miranda.pdf? sequence $=1 \&$ isAllowed $=y$.

17. Martínez H. ¿Son competitivos los Salarios públicos en Colombia? Archivos de economía. [Internet]. Bogotá; D.C; 17 diciembre de 2008. [Fecha de consulta: 04 de septiembre de 2020]. Disponible en: https://www.banrep.gov.co/sites/ default/files/eventos/archivos/martinez_docum_0.pdf.

18. Departamento Administrativo Nacional de Estadística (DANE). Gran encuesta integrada de hogares (GEIH) Mercado laboral [Internet]. Colombia; junio de 2020. [Fecha de consulta 08 de mayo de 2020]. Disponible en: https:// www.dane.gov.co/index.php/estadisticas-por-tema/mercado-laboral/empleo-y-desempleo. 